\title{
EDITORIAL
}

\section{Not Sure if You Are an Investigative Pathologist? Yes, You Are}

\author{
Patricia A. D’Amore, ${ }^{* \dagger}$ Emily H. Essex, ${ }^{\S}$ and Martha B. Furie ${ }^{\llbracket}$
}

From the Schepens Eye Research Institute* of Massachusetts Eye and Ear, Boston, Massachusetts; Departments of Ophthalmology ${ }^{\dagger}$ and Pathology, ${ }^{\ddagger}$ Harvard Medical School, Boston, Massachusetts; the American Society for Investigative Pathology, ${ }^{\S}$ Rockville, Maryland; and the Department of Pathology, ${ }^{\mathbb{}}$ Stony Brook University, Stony Brook, New York

According to The Free Dictionary (https://www. thefreedictionary.com, last accessed November 1, 2021), experimental, or investigative, pathology is: "The scientific study of the nature of disease and its causes, processes, development, and consequences."

To reveal the pathogenesis of most diseases, you first need to understand what is normal. The identification of mutations that drive pathologies may often help reveal the molecular details of normal function. Consider dystrophin, the protein that is mutated in a number of forms of muscular dystrophy. The protein encoded by the mutated gene was identified in 1987, but decades passed before it was demonstrated that dystrophin functions to link muscle fibers to the extracellular matrix via the plasma membrane, stabilizing the sarcolemma during the process of muscle contraction. The scientists who performed this research are investigative pathologists.

Now, think about a recent grant application (NIH or otherwise) and the ever-critical Significance section. What did you cite as the motivation for your studies? Did you write that you want to investigate the structure and function of a certain protein, cell, or organ because it is generally important to understand biology? More than likely, no. Instead, you described how understanding that protein, cell, or organ function will provide insights into the causes of its dysfunction, a step necessary for prevention, diagnosis, management, treatment, or cure.

You may consider yourself a developmental biologist studying the blood-brain barrier, but in a grant application it is quite possible that you would discuss how the blood-brain barrier is reduced in patients at high risk for Alzheimer disease and how understanding the development and maintenance of the blood-brain barrier would illuminate the pathogenesis of this disorder. Or you are a cell biologist who studies the basic structure and function of critical proteins. If you study tubulin stability, your work is relevant to retinitis pigmentosa and cardiac hypertrophy. If you have focused on nuclear lamin A, you are working on the protein whose mutation leads to progeria. You have made a knockout mouse for your favorite protein, and it turns out to have a dramatic pulmonary defect that looks just like a human chronic obstructive pulmonary disease, so you have now generated a model that will be used by others to study pathogenic mechanisms and test therapeutics. All of these examples highlight the work of an investigative pathologist.

Gone are the days of distinct lines between department and fields. A broad range of researchers study how biological processes work and how they can go awry, making us all investigative pathologists. Consequently, almost every researcher in biology can find a home at The American Society for Investigative Pathology (ASIP, https://www. asip.org, last accessed November 1, 2021). ASIP welcomes all investigators who are performing basic or translational research that relates to pathogenesis and mechanisms of disease. Membership in ASIP provides access to a diverse panel of professionals for networking and collaboration at all stages of one's career. ASIP membership also offers career development opportunities that include leadership roles, educational resources, visiting lectureships, and a variety of travel and meritorious awards. There are also financial benefits that come with membership: free and discounted access to scholarly publications and textbooks, discounted rates on meeting registration and continuing medical education programs, as well as reduced rates when publishing in the Society's flagship journal, The American Journal of

Accepted for publication November 3, 2021.

Disclosures: None declared.

Address correspondence to Martha B. Furie, Ph.D., Room 248 Centers for Molecular Medicine, Stony Brook University, Stony Brook, NY 11794 5120. E-mail: martha.furie@stonybrook.edu. 
Pathology (AJP, https://ajp.amjpathol.org, last accessed November 1, 2021).

Just as you may find a welcoming professional home at ASIP, your work may fit well in the pages of AJP. Articles in $A J P$ enjoy excellent visibility. AJP is the mostly highly cited journal in the field, averaging around 43,000 citations per year. Moreover, its articles were downloaded more than 1.8 million times in 2020 alone.

$A J P$ stands apart from most pathology journals, however, due to its focus on mechanistic studies of disease, as opposed to reports that are descriptive or primarily relevant to the clinical practice of pathology. The Journal also welcomes research that generates large data sets that provide a foundation for future mechanistic studies. In 2019, a new topic category was added to encompass application of artificial intelligence and deep learning to issues in pathology, an area where $A J P$ is rapidly becoming a leading forum. ${ }^{1,2} A J P$ 's mission to advance our knowledge of how disease develops is reflected in its statement of scope:

The American Journal of Pathology, official journal of the American Society for Investigative Pathology, published by Elsevier, Inc., seeks high-quality original research reports, reviews, and commentaries related to the molecular and cellular basis of disease. The editors will consider basic, translational, and clinical investigations that directly address mechanisms of pathogenesis or provide a foundation for future mechanistic inquiries. Examples of such foundational investigations include data mining, identification of biomarkers, molecular pathology, and discovery research. Foundational studies that incorporate deep learning and artificial intelligence are also welcome. High priority is given to studies of human disease and relevant experimental models using molecular, cellular, and organismal approaches.

In short, if your research lends insight into causes and progression of disease, it fits the mission of AJP. The Journal's commitment to addressing pathogenesis in a broad sense is reflected in its 17 topic categories (AJP, https://ajp.amjpathol.org/content/authorinfo, last accessed November 1, 2021). Some categories encompass organ systems, such as Cardiovascular, Pulmonary, and Renal Pathology; Gastrointestinal, Hepatobiliary, and Pancreatic Pathology; and Musculoskeletal Pathology. Others address subjects that transcend specific organs, including Genomics, Proteomics, and Gene Regulation; Cell Injury, Repair, Aging, and Apoptosis; Growth Factors, Cytokines, and Cell Cycle Molecules; and Tumorigenesis and Neoplastic Progression.

Does $A J P$ truly embrace contributions from researchers who likely do not regard themselves as pathologists? The answer is absolutely! Of the 12 original research papers published in this January 2022 issue, only four were submitted by corresponding authors affiliated with a Department of Pathology or Pathobiology. The remainder are members of a wide spectrum of departments, including Pharmacology, Immunobiology, Physiology, and Biological Sciences. Each month, two articles are selected as Editor's Choices, the full text of which is made freely available to all readers upon publication. Of the $24 \mathrm{Ed}-$ itor's Choice papers that appeared in 2020, only one-third were submitted by corresponding authors who listed any affiliation with a Department of Pathology. The editors of AJP recognize that research that furthers the understanding of human illnesses is performed in many entities besides traditional pathology departments, and we want to raise awareness that the scope of $A J P$ encompasses work that many investigators might not recognize as "pathology."

Together, ASIP and AJP strive to be destinations for a broad range of researchers to collaborate, investigate, and disseminate their work, and the title of "pathologist" or an affiliation with a traditional Department of Pathology is not a prerequisite for participation. The leadership of ASIP and AJP hopes that all researchers whose work is relevant to mechanisms of disease will consider our society for membership (ASIP, https://www.asip.org/membership-community/join, last accessed November 1, 2021) and our journal for publication of their work (Editorial Manager, https://www. editorialmanager.com/ajpa/default1.aspx, last accessed November 1, 2021).

\section{Acknowledgment}

We thank Dr. Chhavi Chauhan (Director of Scientific Outreach, The American Society for Investigative Pathology) for thoughtful suggestions.

\section{References}

1. Cohen S, Furie MB: Artificial intelligence and pathobiology join forces in The American Journal of Pathology. Am J Pathol 2019, 189:4-5

2. Cohen S, Levenson R, Pantanowitz L: Artificial intelligence in pathology. Am J Pathol 2021, 191:1670-1672 